KATJA MELLMANN

\title{
Biologische Ansätze zum Verhältnis von Literatur und Emotionen
}

In der literaturwissenschaftlichen Emotionsforschung, so ein wahrnehmbarer Konsens, kommt man nicht umhin, transdisziplinär zu arbeiten (Huber 2004, 344; Mellmann 2006a, 12, 14; Anz 2007, 209). Denn will man nicht metaphorisch von »Emotionen in Texten « oder »Affekten der Literatur" sprechen (vgl. die Kritik bei Anz 2007, 212-214), sondern Emotionen als »biopsychisches Phänomen«, das »einen emotionsfähigen Organismus voraus[setzt] « (Mellmann 2006a, 20), ernst nehmen, wird man sich der Instanz des Lesers zuwenden müssen - sei es eines empirischen oder hypothetischen Lesers. `Der Mensch als Instanz im literarischen Kommunikationsmodell wird durch den textwissenschaftlichen Ansatz jedoch nicht mehr erfasst, reale Emotionen sind dem traditionellen philologischen Handwerkszeug unzugänglich. Man kann sich nur mit der Mimesis von Emotionen in Texten befassen und z.B. sagen: »Figur X ist traurig« oder »Figur $\mathrm{X}$ ist eifersüchtig auf Figur Y«; oder man kann sich mit der literarischen Ausgestaltung einer Emotion befassen und z. B. sagen: »Das Gefühl LLieber wird im 18. Jahrhundert mit Naturschwärmerei und dem Gefühl des Unendlichen verbunden«. So weit lässt sich mit textanalytischen Beobachtungen argumentieren. Aber man kann nicht von einer »lustigen Szene« oder einem »traurigen Ausgang", strenggenommen auch nicht von einem »erhabenen Stil« oder von »Unterhaltungsliteratur« sprechen, ohne sich auf die emotionale Wirkung der Texte auf einen Leser zu beziehen. Und diese Wirkung lässt sich nur über die Korrelation bestimmter Textmerkmale mit den Gesetzmäßigkeiten einer reagierenden Leserpsyche bestimmen. Denn was findet ein Leser lustig, traurig, erhaben, unterhaltend - und warum? Hier sind also humanwissenschaftliche Heuristiken gefragt, die über solche Gesetzmäßigkeiten Aufschluss geben.

Die Biologie als eine solche ,Wissenschaft vom Menschen kann nicht auf alle Fragen dieser Art eine ausreichende Antwort geben, aber doch auf einige sehr fundamentale. Das im Folgenden vorzustellende Instrumentarium ist also nicht gedacht, alle offenen Probleme zu lösen und andere Methoden der literaturwissenschaftlichen Emotionsforschung zu ersetzen. Denn es ist z.B. nicht geeignet, individuelles emotionales Erleben vollständig aufzuschlüsseln. Aber es ist hervor- 
ragend geeignet, sehr basale (meistenteils unbewusste) emotionale Reaktionen wissenschaftlich greifbar zu machen und damit die basalen psychischen Strukturen zu identifizieren, auf denen auch eine kulturell komplexe und je individuelle Reaktion noch aufbaut. Eine biologisch argumentierende Psychologie ist immer nur in gegenseitiger Ergänzung mit Sozial- und Individualpsychologie zu denken. Wilhelm Scherers Dreiheit von `Ererbtem`, ‘Erlerntem` und `Erlebtem` erweist sich als ein nach wie vor fruchtbares Forschungsprogramm, wenn es darum geht, menschliches Verhalten (darunter auch literarisches oder Kunst-Verhalten) zu erhellen (vgl. Eibl 2007, 486). Während aber die Kategorie des `Erlebten im Positivismus - und wieder in der psychoanalytischen Literaturwissenschaft - und die Kategorie des `Erlernten von der älteren Einflussforschung über die Ideengeschichte bis hin zur Diskurs- und Begriffsgeschichte starke (manchmal übertriebene) Berücksichtigung gefunden haben, ist die Kategorie des `Ererbten risch unterbelichtet geblieben.

Dies ist kein Versäumnis der Literaturpsychologie allein, sondern Folge desselben Defizits in der zünftigen Psychologie des 20. Jahrhunderts, die vor allem durch die Dominanz des Behaviorismus gekennzeichnet war. »Biologische Psychologie« (vgl. Birbaumer/Schmidt 1999; Schandry 2003) trat in dieser Zeit vor allem als Physiologie auf, d.h. als experimentelle Forschung zu den proximaten Ursachen menschlichen Verhaltens. Mit den ultimaten Ursachen, d.h. mit der evolutionsbiologischen Erklärung von Verhalten, befassten sich verstärkt erst wieder die Soziobiologie und, aus ihr hervorgegangen, die Evolutionspsychologie (Überblick bei Eibl 2004, 47-72). Während die Soziobiologie sich primär am Tierverhalten orientiert und deshalb die Unterscheidung von (aktuell) funktionalem und (prähistorisch) adaptivem Verhalten (s. Tooby/Cosmides 1990) recht lax handhaben und die Ebene des Bewusstseins weiterhin >behavioristisch aussparen kann, widmet sich die Evolutionäre Psychologie nun explizit der Natur des Menschen und den besonderen Bedingungen seines Verhaltens und Erlebens. Damit schließt sie außerdem an die modernen Kognitionswissenschaften an (s. Cosmides/Tooby 1991; 1994; 2003), die als erstes die black box des Behaviorismus wieder aufzuhellen begonnen hatten, und kann auf dem dort bereits eingeschlagenen Weg der funktionalistischen Analyse mit ihrem spezifischeren Bezugsrahmen der biologischen Evolutionstheorie neue Impulse geben (vgl. Mellmann 2006a, 17). Bisherige Versuche einer >darwinistischen Literaturwissenschaft< (Carroll 1995; 2004; Storey 1996; Gottschall/Wilson 2005) sind stärker soziobiologisch als evolutionspsychologisch ausgerichtet. Ähnlich den soziobiologischen Überlegungen zu Kunst und Literatur (Cooke/Turner 1999; Voland/Grammar 2003) befassen sie sich vor allem mit literarischen Inhalten, d.h. mit der Widerspiegelung biologischer Sachverhalte in Literatur oder mit dem unmittelbaren biologischen Nutzen von Literatur als Informationsträger (vgl. die Kritik bei Miall 2001, 408, und Eibl 2007, 488f.). Für das Thema `Emotionen<, verstanden als Leseremotionen, sind diese Studien weniger ergiebig. 
Einschlägig für die folgenden Ausführungen sind eher solche Studien, die den evolutionspsychologischen Ansatz nutzen, um produktions- und rezeptionsästhetische Fragen neu aufzurollen, d.h. die Instanz >Mensch $<$ im literarischen Kommunikationsmodell neu zu beleuchten (s. exemplarisch Thornhill 1998; Tooby/Cosmides 2006; Schwender 2006; Eibl 2004, 277-352; Smith 2005; Zunshine 2006; Voland 2007; und die in diesen Studien verhandelte Literatur; mit Einschränkungen auch Miall 2001; Menninghaus 2003; Boyd 2005). Überlegungen dieser Art befassen sich zum einen mit der Frage, ob Kunst, Literatur, Erzählen usw. Adaptationen (Anpassungen) oder evolutionäre Nebenprodukte sind, und zum anderen mit der Ausformulierung einer sevolutionären Ästhetikı, d.h. mit der Frage, was wir svon Natur ausı schön finden und warum. Diese Forschungen decken vor allem denjenigen Bereich des Ästhetischen ab, der im Sinne Kants als das Angenehme kategorisiert werden könnte, also Phänomene der Lust und der angeborenen Präferenzen. Im spezifischeren Sinne emotionale Reaktionen auf Literatur und Kunst sind seltener Gegenstand einschlägiger Studien gewesen. Hervorzuheben sind vor allem zwei filmwissenschaftliche Beiträge zu Rührung und Humor (Tan/Frijda 1999; Eitzen 1999), auf die ich später noch ausführlicher zu sprechen komme und die meines Erachtens wegweisend sind für das, was eine evolutionspsychologisch informierte Emotionsforschung in den Literaturwissenschaften leisten könnte: nämlich

a) die evolutionstheoretische Plausibilisierung einer distinkten Emotion (eines bereichsspezifischen psychischen >Moduls<; vgl. Tooby/Cosmides 1992, 73-77) b) und die Postulierung eines zugehörigen auslösenden Reiəschemas, das sich an den Texten (Filmen, sonstigen medialen Darstellungen) verifizieren lässt.

Wenn die erwartbaren physischen und kognitiven Begleitvorgänge der Emotion und das textuelle Reizschema ausreichend konkretisiert werden, können so gebildete Reiz-Reaktions-Hypothesen zudem dem Verfahren empirischer Testung unterzogen werden. Dies allerdings nicht im Sinne einer deterministischen Wenn-dann-Aussage, die sich auf das emotionale Erleben im vollen Umfang erstreckt. Ich werde im folgenden ersten Abschnitt zeigen, dass die reflexhaft zzwingend einsetzende emotionale Reaktion sich meistenteils auf einige wenige basale physische Vorgänge (z.B. Veränderung der Herzfrequenz, des Gesichtsausdrucks) beschränkt; emotionsgesteuerte Kognitionen, wie sie als `Gefühk in die bewusste Selbstwahrnehmung eingehen, und erst recht Handlungsresultate hängen hingegen von einer Reihe von kulturellen und individuellen Umständen ab, die in einer experimentellen Überprüfung mit zu berücksichtigen wären (vgl. Mellmann 2006a, 82f.).

Die folgende Einführung in biologische Ansätze zum Verhältnis von Literatur und Emotionen fußt also auf zwei thematischen Eingrenzungen: Unter Emotionen werden emotionale Reaktionen auf Literatur, nicht literarische Präsentationen von Emotionen verstanden; als biologische Ansätze werden in erster Linie evolutionspsychologische Ansätze berücksichtigt. Ich erläutere zunächst das 
emotionstheoretische Modell, das einer literaturwissenschaftlichen Emotionsforschung zugrunde gelegt werden kann (1). Anschließend wird die Frage behandelt, was uns überhaupt dazu motiviert, uns der emotionalen Wirkung literarischer Kunstwerke auszusetzen (2). Der darauffolgende Abschnitt behandelt am Beispiel der Landschaftsästhetik die literarische Verwendung von Annäherungs- und Abstoßungsreizen (3). Als zwei Beispiele spezifischen emotionalen Verhaltens werden dann die Reaktionen Lachen und Weinen besprochen (4). Im letzten Abschnitt werden verschiedene Sympathiegrade und deren Auswirkungen auf unser soziales Bindungsverhalten gegenüber Kunstwerken vorgestellt (5).

\section{Evolutionspsychologische Emotionstheorie und literarischer Rezeptionsprozess}

Verlässt man die textimmanente Analyse literarischer Emotionsdarstellungen zugunsten einer Untersuchung von Leseremotionen, kommt man nicht umhin, den Rezeptionsprozess nach dem ethologischen Schema von Reiz, und Reaktion zu modellieren (vgl. auch Anz 2007, 214-217). Der Text - bzw. die durch ihn beim Leser evozierte Imagination - wird als Stimulus, die genetisch svorprogrammierte Leserpsyche als Reaktionsinstanz verstanden. Die heuristisch anzunehmende Reaktionsinstanz lässt sich konzipieren als eine Art »anthropologische[r] Modell-Leser«, der keine individuelle oder statistisch generalisierte Psyche, sondern das »Ensemble aller psychischen Grundfunktionen« repräsentiert, die sich evolutionspsychologisch postulieren lassen (Mellmann 2006a, 21).

Die Reaktionen auf medial repräsentierte Inhalte sind in evolutionspsychologischer Perspektive allerdings weitgehend als Attrappenwirkungen zu verstehen (Mellmann 2006a, 42-78; 2006b; vgl. auch schon Schwender 2006). Denn die medialen (১fiktionalen`) Reize, auf die unser emotionaler Apparat reagiert, sind in der Regel nicht identisch mit denjenigen Umweltreizen, an denen sich die jeweilige Emotion in der Umwelt unserer Vorfahren (im EEA, dem »environment of evolutionary adaptedness«; vgl. Tooby/Cosmides 1990, 386-388) herausgebildet hat, sondern Artefakte, auf die unsere ererbten psychischen Mechanismen gewissermaßen sirrtümlich` (Konrad Lorenz; vgl. Mellmann 2006a, 36) ansprechen. Dass wir z. B. über ein Emotionsprogramm >Mitleid (hier: misericordia; vgl. Mellmann 2006a, 124ff.) verfügen, lässt sich vermutlich darauf zurückführen, dass die Vorfahren des Menschen in Gruppen zusammengelebt haben und eine helfend-schützende Reaktion auf die Hilflosigkeit eines (in der Regel verwandten) Gruppenmitglieds im Sinne der sinklusiven Fitness` (s. Eibl 2004, 53) eine verstärkte Reproduktion der eigenen Gene zur Folge hatte. Wenn nun aber ein weinendes Gretchen oder eine verweinte, klagende, von Selbstvorwürfen zermürbte Sara Sampson unser Mitleid erregen, dann spricht unser emotionaler 
Apparat auf evolutionär irrelevante Reize (〉Attrappen`) an, die lediglich dem evolvierten emotionsspezifischen Reizschema entsprechen.

Die entwicklungsgeschichtliche Perspektive und das Attrappen-Modell erweisen sich als hilfreich, das sogenannte "paradox of fiction« aufzulösen (vgl. Mellmann 2006a, 59-68; 2006b). Colin Radford stellte 1975 in seinem Aufsatz »How Can We Be Moved by the Fate of Anna Karenina? « fest, dass eine emotionale Reaktion auf Fiktionen zwar eine Tatsache, aber irrational sei. In radikal kognitivistisch argumentierenden Beiträgen (exemplarisch Levinson 1997) zum Thema bereitet diese Tatsache bis heute Schwierigkeiten, da angenommen wird (warum?), dass emotionale Reaktionen auf einen Gegenstand logisch die Überzeugung von der Existenz dieses Gegenstands impliziere. Wenn die Autoren aber alle bekunden, dass das Schicksal Anna Kareninas, Little Nells oder welcher Figuren auch immer sie emotional bewege, dann haben wir gewissermaßen eine inoffizielle Versuchsreihe vor uns, die belegt, dass diese Prämisse falsch ist; d.h. die belegt, dass die Attrappe >Romanfigur in ausreichendem Umfang dieselben auslöserelvanten Merkmale zeigt wie wirkliche Personen und die Frage der Faktizität in diesen Auslöseschemata offenbar keine Rolle spielt. Die Unterscheidung von >realer und >virtueller Erfahrung wird »zwar rational, kaum aber affektiv vollzogen« (Voland 2007, 8). Denn insbesondere Emotionen gehorchen weitgehend den »intuitiven Ontologien« unseres psychischen Apparates, die »durch die natürliche Selektion so gestaltet worden [sind], dass sie adaptive Probleme lösen helfen«; und »[n]icht jene Gehirne gingen aus dem survival of the fittest hervor, die die Regeln der Logik und Rationalität am besten beherrschten, sondern diejenigen, die mit der Welt am nützlichsten umgehen konnten« (Voland 2007, 9). Gerade die Irrationalität eines Verhaltens, wie etwa Radford es am Verhalten gegenüber Literatur beobachtet hat, kann ein Hinweis sein, dass eine begriffslogische Analyse des Sachverhalts nicht weiterführt und man zur Aufklärung einer externen Argumentationsbasis bedarf. Die biologische Evolutionstheorie ist eine solche externe Argumentationslogik, und sie gibt Aufschluss über den evolutionären `Sinn` und `Unsinn`, die adaptive `Rationalitätı, eines uns heute irrational erscheinenden Verhaltens (Beispiele bei Mellmann 2006a, 63-69).

Da wir aber, wie schon Radford bemerkte, nicht ganz und gar gleich, sondern nur ähnlich auf fiktionale und wirkliche Reize reagieren, muss zu der Erklärung emotionaler Literaturwirkungen als Attrappenwirkungen noch etwas hinzukommen: Emotionen können zwar durch fiktionale und reale Stimuli gleichermaßen ausgelöstwerden, dann aber jeweils einen ganz unterschiedlichen Verlauf nehmen. Denn der Verhaltensmodus der Emotion zeigt (im Unterschied zu dem des Reflexes) keine strikte Kopplung von Auslösereiz und Verhaltensreaktion, sondern eine Verzögerungsspanne zwischen beidem, in der eine zweite Situationseinschätzung stattfinden und das Verhalten angepasst werden kann (Scherer 1994). Diese zweite Situationseinschätzung gehorcht einem jeweils emotionsspezifischen Programm, d.h. die weitere Informationsaufnahme unterliegt einem für 
die ausgelöste Emotion spezifischen Selektionsraster, das ganz bestimmte, für die emotional programmierten Verhaltensoptionen relevante Informationen abfragt und je nach Situationsfeedback bestimmte Anpassungen des Verhaltens vorsieht (Cosmides/Tooby 2001).

Das kann im Fall von fiktionalen Auslösern zu einem schnellen Beenden des Emotionsprogramms führen, zumal im Fall von energieaufwendigen Stressemotionen. Es ist nicht anzunehmen, dass der Leser einer Gespenstergeschichte sich dauerhaft und buchstäblich im Zustand der Angst befindet, denn wovor sollte er sich fürchten? Die Programme von Furchtemotionen dürften nach Maßgabe der Evolution adaptiver Mechanismen so gebaut sein, dass der Auslösemechanismus zwar svorsichtshalber< relativ leicht anspringt (vgl. Voland 2007, 13f.), der Verlauf der Emotion dann aber bei Nichtakutheit des Reizes (z. B. im Fall fiktionaler Stimuli) abgekürzt, die Emotion schnell wieder beendet wird, um unnötige Belastungen des Körpers durch die Ausschüttung von Stresshormonen und die damit verbundenen physischen Begleiterscheinungen möglichst gering zu halten. Ein geschickter Autor wird freilich dafür sorgen, dass immer wieder neue Reize dargeboten und Furchtemotionen immer wieder aufs Neue ausgelöst werden. Begriffe wie »thrill« oder "gruselig« bezeichnen sehr anschaulich das Auf und $\mathrm{Ab}$ von Emotionsauslösung und Programmbeendigung, wie es uns gegenüber Texten des Horror-Genres (etwa von Stephen King) vertraut ist. In Emotionsprogrammen, die keine große Belastung für den Körper darstellen, ist hingegen nicht mit einer so rigiden Anpassung an die Fiktionalität des Reizes zu rechnen. Dies scheint z.B. der Fall zu sein bei den emotionalen Reaktionen auf Romane mit erotischem oder pornographischem Inhalt. Die Attraktivität pornographischer Literatur besteht ja gerade darin, dass sie dieselben angenehmen Gefühle im Leser hervorruft wie tatsächliche Situationen sexueller Intimität. Auch die vielen sozialen Emotionen (Mitleid, Bewunderung, Verachtung, Sympathie, Liebe, Hass, ...), mit denen wir auf literarische Figuren reagieren, sind dem Erleben im wirklichen Leben sehr ähnlich. Sie werden durch die Mimesis menschlichen Verhaltens in diesen Figuren ausgelöst und setzen eine Reihe emotionsspezifischer kognitiver Algorithmen in Gang. Der Leser ist, ohne dass er sich dafür besonders anstrengen müsste, stets bestens informiert darüber, warum Figur A so unglücklich ist; was Figur B tun könnte, um die Situation zu ändern; was die einzelnen Figuren wissen oder nicht wissen; usw. Nahezu unser gesamtes Handlungsverstehen ist emotionsgeleitet. Allerdings wird im Normalfall keine sirrationale aktive Verhaltensreaktion ( $\gg$ Affekthandlung`) der Attrappe gegenüber erfolgen - wie etwa ein zischendes »Du Mistkerl!« adressiert an den Bösewicht (aber auch das mag im Einzelfall vorkommen) oder gar ein tätlicher Angriff (wie er im Kasperltheater mitunter auftaucht).

Der Standardfall emotionaler Reaktionen gegenüber fiktionaler Literatur dürfte also sein, dass wir zwar eine kognitive Aktivierung und in gewissem Umfang auch körperliche Veränderungen erleben, äußerlich aber inaktiv bleiben: 
Obwohl Fiktionen wie Attrappenwirkungen verarbeitet zu werden scheinen, reagieren einige psychische Subsysteme verläßlich so auf sie, als wären sie Wirklichkeit, während andere dies verläßlich nicht tun. [...] Eine spannende Abfolge fiktionaler Ereignisse wird ein umfangreiches Spektrum emotionaler Reaktionen aus unseren gesamten mentalen Mechanismen auswählen - und zwar dieselben Reaktionen, die solchen Ereignissen und Personen auch in der Wirklichkeit zukämen. Wir empfinden etwas für die beteiligten Figuren, wir machen unser Wohlbefinden von einer oder mehreren von ihnen abhängig, wir können Furcht, Abscheu oder Erschütterung empfinden, so als würden diese Ereignisse uns selbst zustoßen. Wir empfinden tief, handeln aber nicht im geringsten $[\ldots]$.

(Tooby/Cosmides 2006, 221)

Um dieser Doppelgesichtigkeit von unterschiedsloser Auslösung einer Emotion und anders geartetem Verlauf gegenüber fiktionalen Szenarien analytisch gerecht $\mathrm{zu}$ werden, habe ich eine Unterscheidung von »Auslösemechanismus« und »Verlaufsprogramm« einer Emotion vorgeschlagen (Mellmann 2006a, 32f.). Der Auslösemechanismus springt an, wenn das zu ihm gehörige Auslöseschema einen >Treffer erzielt, und setzt erste physische Reaktionen in Gang, die den Körper auf eine emotionsspezifische Verhaltensreaktion vorbereiten (z.B. >Herzklopfen als Symptom einer Energiemobilisierung in Vorbereitung auf eine Kampf- oder Fluchtreaktion; sexuelle Erregung u. Ä.). Außerdem initialisiert er das Verlaufsprogramm, das eine spezifisch ausgerichtete Situationsanalyse vornimmt (z.B. Aufmerksamkeit auf Situationsmerkmale, die zu einer Besserung der Lage des Bemitleideten dienen können; Überprüfung der Faktualität des Stimulus bei energieaufwendigen Emotionen). Die neuronalen Rückmeldungen der physischen Begleitvorgänge und die Kognitionen der näheren Situationsanalyse ergeben zusammen das, was als subjektives `Gefühk im Bewusstsein auftaucht.

Das Verlaufsprogramm einer Emotion als Entkoppelung von Stimulus und Verhaltensreaktion ist nicht der einzige Punkt, an dem eine Flexibilisierung des Verhaltens eintreten kann. Außerdem sind zu berücksichtigen die >Reaktion auf die Reaktion<, d.h. unsere affektive Einschätzung eines an uns selbst wahrgenommenen emotionalen Verhaltens, und die kognitive Weiterverarbeitung oder Bewältigung dieses Befundes und, damit zusammenhängend, die bewusste Wahl einer Verhaltensstrategie (Mellmann 2006a, 79-85). Dies betrifft insbesondere die Kontrolle unseres Ausdrucksverhaltens. Einem still versunkenen Leser eines spannenden Romans sieht man die Spannung an Gesicht und Körperhaltung durchaus an, und beim tragischen Tod der Geliebten des Helden mag er vielleicht sogar einmal nasse Augen bekommen. Unter Beobachtung aber, z.B. im Theater sitzend, sind ihm Tränen vielleicht peinlich und er versucht sie zu unterdrücken. Werden Tränen hingegen kulturell valorisiert wie z.B. im Gefühlskult der Empfindsamkeit, mag er ihnen gerade in einer solchen Situation umso freieren Lauf lassen. Anders als bei der Verhaltensflexibilisierung durch das Verlaufsprogramm bewegen sich die >Reaktion auf die Reaktion Bewältigungsverhalten nicht mehr innerhalb des durch die jeweilige Emotion sorprogrammierten Anpassungsspektrums, sondern können verstärkt auch 
remotionsexterner mentale Faktoren in Acht nehmen und rationale Verhaltensplanung miteinbeziehen. Nicht alle Verhaltenskomponenten aber unterliegen im gleichen Maße unserer Kontrolle. Insbesondere Gesichtsausdrücke und Verhaltensreaktionen aus dem Bereich des Lachens und Weinens bzw. deren Vorbereitungssymptome wie das `Glucksen (als Ansatz eines Lachens) oder der ২Kloß im Hals` (als Ansatz eines Schluchzens) sind willentlich nur schwer zu unterdrücken. Denn Lachen und Weinen sind Reaktionen aus dem Bereich unserer sozialen Dispositionen, für die eben gerade die expressive Displayfunktion (Lachen als Kooperations- und Spielangebot, Weinen als Signal von Hilfsbedürftigkeit) zentral ist.

Bisher habe ich Emotionen ausschließlich als angeborene Mechanismen behandelt. Es ist jedoch bekannt, dass es auch zahlreiche ssozial vermittelte< Emotionen gibt. Wovor wir uns ekeln oder wofür wir uns schämen, sagt man z.B. häufig, sei anerzogen. Die Emotionen Ekel und Scham selbst aber sind angeborene psychische Programme. Dass wir über diese natürlichen Dispositionen verfügen, ermöglicht überhaupt erst, dass wir im Laufe der Ontogenese auf weitere Auslösereize konditioniert werden können. Gerade Kunst wirkt an solchen ontogenetischen Prägungen ganz erheblich mit. Der Sternenhimmel z. B. ist biologisch gesehen ein neutraler Reiz. Von ihm geht keinerlei Selektionsdruck auf den Menschen aus. Als literarisches Motiv aber taucht er oft als `erhabener Reiz auf. Die Voraussetzung dafür ist, dass er durch Kunst und Wissenschaft als Symbol des Unendlichen semantisiert wurde und dadurch als Attrappe für unsere Furcht vor unabgeschlossenem Raum taugt (vgl. Mellmann 2006a, 92-94). Die evolutionspsychologische Rede von »angeborenen Programmen« meint nicht, dass ein Programm von Geburt an präsent und bis zum Tod unverändert funktionieren muss, sondern dass es ein genetisch vorprogrammiertes Grundmuster gibt, das ontogenetisch erst entfaltet werden muss, und zwar in Feinabstimmung mit der jeweils ontogenetisch anzutreffenden Umwelt. Dies erklärt nicht nur, warum es trotz des anzunehmenden >Allgemeinmenschlichen zu enormen interkulturellen und interindividuellen Unterschieden kommt, sondern auch, warum wir uns mit so nutzlosen Dingen wie Kunst und Literatur befassen: Denn wenn wir darauf angewiesen sind, dass unsere angeborenen Mechanismen sich im Laufe der Ontogenese selbst zusammenbauen, dann müssen Anlässe dafür geschaffen werden, diese Mechanismen wiederholt zu aktivieren und auf diese Weise nach und nach die nötigen Gehirnverknüpfungen aufzubauen. Literatur ist solch ein Anlass, (vor allem kognitive und emotionale) Adaptationen gefahrlos auszutesten und weiterzuentwickeln. Und zu einer solchen quasi sübenden Betätigung angeborener Mechanismen, wo immer sich Gelegenheit dazu bietet, werden wir motiviert durch Lust. 


\section{Funktionslust}

»Lust« als psychologischer Terminus (s. Frijda 2000) bezieht sich auf die Ausschüttung dopaminerger Neurotransmitter im Gehirn, mit der uns die Evolution für bestimmte, in ihrem Sinne snützlicher Tätigkeiten >belohnt،. Das »mesolimbische Belohnungssystem《 wurde seit seiner Entdeckung 1954 durch die kanadischen Hirnforscher James Olds und Peter Milner (vgl. Schandry 2003, 458f.) vor allem im Kontext von Suchtverhalten erforscht. Die Rolle, die es bei der Steuerung ganz alltäglichen oder speziell kunstbezogenen Verhaltens spielt, war erst vereinzelt Gegenstand neurologischer Studien. Ausschlaggebend für die Entdeckung der Lust als eines generell wichtigen Movens für menschliches Verhalten waren nicht so sehr empirische Beobachtungen als vielmehr der evolutionsbiologisch begründete Verdacht, dass das Belohnungssystem sich aus einem bestimmten Grund entwickelt haben muss: nämlich um adaptives Verhalten zu motivieren und ontogenetisch zu entwickeln.

Die ontogenetische Entfaltung angeborener Antriebe und Fähigkeiten, d.h. der Aufbau und die lebenslange Organisation des Gehirns, stellt nach Ansicht der Evolutionspsychologen John Tooby und Leda Cosmides selbst ein zentrales Anpassungsproblem dar, das mit der Evolution eines eigenen adaptiven Systems beantwortet wurde, das man auch als `Spieltriebく bezeichnen könnte. Die Attraktivität mancher zweckloser Gegenstände, pragmatisch entlasteter Situationen und überschüssiger Gedankenspiele rührt in ihren Augen daher, dass solche Gegenstände, Situationen und virtuellen Konstrukte geeignet sind, unsere Gehirnfunktionen aufzubauen und feinabzustimmen. Um sicherzustellen, dass wir die Gelegenheiten für solch ein gefahrlos-spielerisches Ausprobieren nicht verpassen, sollte »jede psychische Adaptation, von der Sprachfähigkeit bis zum Gehörsinn, mit ihrem eigenen System ästhetischer Präferenzen ausgestattet sein. Denn das Individuum muss zum Erwerb von Erfahrungen motiviert werden, die das entsprechende neurokognitive System zu entwickeln, kalibrieren und abzustimmen helfen« (Tooby/Cosmides 2006, 229).

Solche rästhetischen` Präferenzen zum Erwerb von Erfahrungen werden in der Literatur durch die Herstellung virtueller Erfahrungswelten bedient, die eine strukturelle Isomorphie mit tatsächlichen Erfahrungswelten zeigen (Tooby/ Cosmides 2006, 237). So werden z. B. die Präferenzsysteme unserer zahlreichen sozialen Adaptationen im Figurenrepertoire fiktionaler Erzählungen fündig: Wir haben eine Vorliebe für Texte, die uns Personen anbieten, über die wir nachdenken, um die wir uns empathisch bemühen, die wir mögen oder ablehnen können. Auch für zahlreiche kognitive Adaptationen aus dem Bereich der Gestaltwahrnehmung, an Hand derer das Gehirn Ordnung in die Welt bringt, bietet Literatur immer wieder bevorzugte Objekte an; z.B. Geschichten, die auf dem Schema von Trennung und Wiedersehen, Aufstieg und Fall oder Unrecht und Wiedergutmachung aufbauen; oder die Rätsel anbieten, die gelöst werden müssen, oder 
dräuende Gefahren vorführen, die es abzuwenden gilt. Der ganze Bereich des Nachvollzugs von 'Spannungsbögen kann als hochgradig lustprämiert gelten (zu sSpannungr s. Mellmann 2007). Aber auch z. B. Wortspiele, kausale Handlungsentwicklung, Aporien oder einfach Neugier weckende Antizipationen stellen Reize für vielfältige kognitive Algorithmen zur Verfügung. Lust verursachen nicht zuletzt auch bestimmte Formkriterien von Literatur wie z.B. Reim und Metrum, epische Dopplung und die Dreimaligkeit im Märchen, die unserem Erkennen von Wiederholungen, Übereinstimmung und Abweichung, Symmetrie, Verlaufsgestalten u. Ä. in die Hände spielen.

Dieser weitläufige Bereich der Leselust durch die optimale Bedienung kognitiver Kompetenzen könnte als »Kognitionslust« (Eibl 2004, 340) oder, im Anschluss an Charlotte und Karl Bühler, als »Funktionslust« (vgl. Frijda 2000, 81 f.) bezeichnet werden. Gemeint ist ein unbewusster positiver >Begleitkommentar< (Wilhelm Wundt; vgl. Frijda 2000, 73), der mit der Ausübung angeborener Mechanismen einhergeht - vor allem in pragmatisch entlasteten Situationen oder bei Erfüllung programmeigener Optimalitätskriterien (Frijda 2000, 84-88) und dafür sorgt, dass diese Ausübung möglichst nicht unterbrochen wird (»flow «-Erfahrung). Jedenfalls solange keine sonstigen Situationsanforderungen es nötig machen. Diese Lustkomponente aller evolutionär adaptiven Programmausübungen erklärt nicht nur, warum wir überhaupt gerne solchen nutzlosen Beschäftigungen wie der Romanlektüre nachgehen, sondern auch so scheinbar paradoxe Phänomene wie die `Lust der Tränen oder das sangenehme Grauen`. Denn auch solche snegativen Reaktionen wie die des Weinens und der Furcht basieren auf biologischen Verhaltensdispositionen, deren sübender Ausführung im »Organisationsmodus« (Tooby/Cosmides 2006, 230) also an das dopaminerge Belohnungssystem gekoppelt ist. Entsprechende Auslösereize bereiten also Vergnügen und können deshalb aktiv aufgesucht werden.

Ein anderer Bereich lustprämierter Präferenzsysteme (von »aesthetics« im evolutionspsychologischen Jargon) betrifft unsere Vorliebe für bestimmte Reize und die Abneigung gegenüber anderen; also das, was traditionell als Verhaltenssteuerung durch $\gg$ Lust und Unlust in der Anthropologie firmiert.

\section{Appetitive und aversive Reize}

Der Neuropsychologe Jeffrey A. Gray hat für das Säugetiergehirn drei basale Verhaltenssysteme postuliert, die sich durch distinkte neuronale Erregungsmuster nachweisen lassen und mit unterschiedlichen Verhaltensdispositionen und Reizarten korrelieren (vgl. Birbaumer/Schmidt 1999, 648-657; Mellmann 2006a, 245-247). Das »Annäherungssystem« ist zuständig für Verhaltensweisen der gerichteten Aktivierung (z.B. Beuteaggression, gezielte Flucht) und korreliert mit Annäherungs- oder appetitiven Reizen; das »Kampf/Flucht-« und das »Ver- 
haltenshemmungssystem« sind zuständig für ungerichtete Aktivierung (z. B. Davonlaufen, Verteidigungskampf) und passives Vermeidungsverhalten (z.B. stilles Abwarten); sie korrelieren mit Abstoßungs- oder aversiven Reizen. Diese appetitiven und aversiven Reizqualitäten können prinzipiell sowohl angeboren als auch konditioniert (erlernt) sein. Gerade in der Kunst aber zeigen sich oftmals idealisierte Reizkonfigurationen, die auf angeborenen Präferenzsystemen aufzubauen und daher ihre überhistorische Plausibilität zu beziehen scheinen. Dies sei am Beispiel des locus amoenus und des locus terribilis exemplifiziert.

Neben unseren Präferenzen für bestimmte Gesichts- und Körpereigenschaften im Kontext der Geschlechtspartnerwahl sind es vor allem die Präferenzen für Landschaftseigenschaften, die in der Evolutionären Ästhetik bislang verstärkte Berücksichtigung gefunden haben (s. Orians/Heerwagen 1992; Thornhill 1998). Solche Präferenzen für halboffne Raumstrukturen (vorne Sicht, hinten Schutz), üppiges Grün, die Gegenwart von Wasser, fruchttragenden Pflanzen und essbaren Tieren, die Möglichkeit von Schatten usw. lassen sich aus der Notwendigkeit für unsere Vorfahren erklären, geeignete Habitate zu identifizieren: Eine Anzahl von Umwelteigenschaften, die Sicherheit und Nahrung versprechen, sind für uns einfach attraktiv, schön, angenehm, ohne dass wir über ihre Eignung für unsere vegetativen Bedürfnisse eigens nachdenken müssten. Ein Hinweis darauf, dass es sich um angeborene Präferenzen handelt. Die stereotype Ausstaffierung des `Lustorts mit Gras, schattenspendendem Laub, sprudelnder Quelle und Vogelgezwitscher - und vielleicht noch mit einer Herde Schafe im Hintergrund stellt eine optimale Attrappe gemäß diesen angeborenen Präferenzen dar und erfreut sich demzufolge einer besonderen Langlebigkeit als Bildmotiv in Literatur und Malerei. Evolutionspsychologisch wenig erforscht sind demgegenüber entsprechende aversive Reize, wie sie sich im Bildtopos des locus terribilis oder locus desertus ablesen lassen und wie ich sie unter dem Titel der »vegetativen Dysfunktionalität« gesammelt habe, um einen typischen Motivfundus des `Erhabenen biologisch zu begründen (Mellmann 2006a, 237-240). Reize wie Kargheit, Verlassenheit, Kälte, unabgeschlossener Raum u. Ä. sind Reize, die durch das Ausbleiben von Lust allein $\mathrm{m}$. E. nicht ausreichend spezifiziert wären, sondern denen vielmehr eine eigene emotionale Qualität als Furcht- oder Abstoßungsreize zugesprochen werden kann. Als solche setzen sie ein Vermeidungsverhalten in Gang, das auch als emotionale Wirkung von literarischen Attrappen eine Rolle spielt. Solche durch die Habitatwahl evolutionär begründbaren aversiven Reize wären den Motivbereichen von Kants »mathematisch « und »dynamisch «Erhabenem (der »perzeptiven« und der »motorischen Dysfunktionalität«, vgl. Mellmann 2006a, 233-237) noch hinzuzufügen, wenn man die literarische Präsentation aversiver (potentiell serhabener`) Kulissen wirkungsästhetisch analysieren will.

Die Begrifflichkeit von appetitiven und aversiven Reizqualitäten ist außerdem geeignet, die problematische Entgegensetzung von spositiven Emotionen durch eine spezifischere, nämlich die jeweils korrelierende Verhal- 
tensdisposition (Annäherung oder Vermeidung) benennende Unterscheidung zu ersetzen. Die Rede von 'positiven und `negativen halb problematisch, weil auch inegativer Emotionen von Funktionslust begleitet sein können (`Lust der Tränen〈, rangenehmes Grauen`), und zudem deshalb, weil sie kulturabhängig und individuell auf der Ebene der >Reaktion auf die Reaktion im Einzelfall recht unterschiedlich bewertet werden können (s. Mellmann 2006a, 79-83). Das jeweils subjektive Erleben einer Emotion (als spositiv $<$ oder snegativ $\iota)$ ist in einer evolutionspsychologischen Perspektive weniger ausschlaggebend als die Bereichsspezifik des jeweiligen Programms, d.h. die Frage, welchem Selektionsproblem ein bestimmtes psychisches `Moduk jeweils zuzuordnen ist. Auch die nun folgenden Ausführungen zu den Reaktionen des Lachens und Weinens sind in diesem Sinne also nicht zu verstehen als Beispiele für eine besonders positive und eine besonders negative emotionale Erfahrung, sondern als zwei Beispiele für angeborene Verhaltensmechanismen, die sich als Reaktionen auf spezifische, statistisch wiederkehrende selektionsrelevante Situationen im EEA entwickelt haben.

\section{Lachen und Weinen}

Der Filmwissenschaftler Ed Tan und der Psychologe Nico Frijda haben in einem 1999 erschienenen Aufsatz versucht, das Reizschema für die Reaktion des Weinens auf ssentimentale` (rrührende`) Geschichten in Literatur und Film näher zu bestimmen. Sie argumentieren nicht strikt evolutionspsychologisch, beziehen sich aber (ebenso Koch 2004) auf die Anthropologie Helmuth Plessners, die hierzu einige Anschlüsse bereitstellt. Die Reaktion des Weinens ist nach Tan und Frijda nicht so sehr ein Kennzeichen der Emotionen Kummer und Trauer, wie die Alltagspsychologie nahelegt, sondern vielmehr eine Reaktion auf Situationen der Überforderung und Ohnmacht. Als Auslösereiz in narrativen Fiktionen, der dieses Schema erfüllt, machen sie »any major resolution in a conflict« (Tan/ Frijda 1999, 54) fest, d.h. jede resultative Handlungsentwicklung, die dem handlungsbegleitenden >Hoffen und Bangen des Rezipienten und seinen kognitiven Bewältigungsbemühungen ein plötzliches Ende setzt, so dass er erkennen muss, dass inichts mehr auszurichten ist. Man könnte auch sagen: am Ende eines Spannungsbogens (Mellmann 2006a, 132; 2007, 264f.; Koch 2004, 569). Dies gilt sowohl für spositiver als auch für snegativer Entwicklungen der Handlung: Nicht nur Szenen am Sterbebett, eine verhängnisoffenbarende Anagnorisis, der Tugendverlust oder das Scheitern eines Helden verursachen Rührung, sondern auch seine Aufnahme in die Gemeinschaft, die Hochzeit am Ende eines Romans, der Kuss am Ende eines Films, die Geburt eines gesunden Kindes oder der >Triumph der Tugendk. Notwendige Bedingung ist vielmehr, dass die beteiligten Emotionen existentielle menschliche Bedürfnisse tangieren. Tan und Frijda 
nennen als Beispiele für solche existentiellen Bedürfnisse den Bereich des sozialen Gerechtigkeitsdenkens (Schema `Gut/Böse`), der Selbsterhaltung (Schema ,Überleben/Sterben`) und des sozialen Bindungsverhaltens (Schema `Trennung/ Wiedervereinigung $)$.

$\mathrm{Zu}$ den physischen Begleiterscheinungen einer solchermaßen ausgelösten Kapitulationsreaktion in Situationen der Überforderung und Ohnmacht gehören neben dem wohlbekannten $>K l o ß$ im Hals und dem Tränenvergießen auch das /Weichwerden', d.h. eine allgemeine Muskelerschlaffung (Mellmann 2006a, 81, 131; Koch 2004, 572), die an die Demutsgesten anderer Säugetiere erinnert. Solche Unterwerfungsgesten haben ihren adaptiven Sinn in der Signalisierung von Hilfsbedürftigkeit, wie sie in Situationen der nichtparierbaren Bedrohung, bei verlorenem Anschluss an die Herde oder Verlust eines Caretakers im EEA überlebensförderlich gewesen sein mag. Die sentimentale Rührung - als Kapitulationsreaktion auf eine Attrappe - ist demgemäß mit dem subjektiven Empfinden eines Autonomieverlustes (Tan/Frijda 1999, 55) verbunden, der auf der Ebene der >Reaktion auf die Reaktion` z. B. dafür sorgt, dass Frauen - gemäß den kulturell gültigen Genderrollen - das Gefühl der Rührung und die begleitenden Tränen eher zulassen als Männer.

Im selben Band, in dem der Artikel von Tan und Frijda erschienen ist, hat der Filmwissenschaftler Dirk Eitzen dafür plädiert, Humor als eine angeborene Disposition anzusehen, die in Situationen von gelindem sozialen Stress Kooperationsbereitschaft signalisiert (Eitzen 1999, bes. 93-99; jetzt auch Gervais/Wilson 2005). Das bedeutet, dass Lachen erstens hochgradig funktionslustprämiert ist und zweitens mit der Imagination (mit dem »psychopoetischen Effekt «, vgl. Mellmann 2006a, 99-103) eines scherzenden sozialen Gegenübers (z.B. eines ironischen oder spöttelnden Erzählers, einer witzigen Figur oder eines parodistischen lyrischen Sprechers) einhergeht. Das Auslöseschema der Lachreaktion, ssozialer Stressı unter der Rahmenbedingung sSicherheit, habe ich in Anwendung auf literarische Texte als Technik des sunschwerwiegenden Fehlers zu spezifizieren versucht, der den Rezipienten in eine stresshafte Kommunikationssituation versetzt: Verstöße gegen die Sprachlogik (z.B. Verstöße gegen die semantische Kombinationslogik oder die Textkohäsion wie in Wortspielen, uneigentlicher Rede, Ambiguitäten, Überraschungen und Erwartungsbrüchen) oder gegen sittliche Normen (z.B. Verstöße gegen das Decorum, derbe Komik oder verhaltene Aggressivität) können den Leser zum Lachen reizen, sofern er sich nicht selbst persönlich betroffen, d.h. vom Kooperationsangebot des scherzenden Gleichgesinnten ausgeschlossen fühlt (Mellmann 2006a, 275f., 332-338). An dieser Beschreibung wird bereits offensichtlich, dass die lachende Reaktion ebenso universal (wir alle lachen auf dieselben strukturellen Effekte hin) wie auch von individuellen und kulturell spezifischen Bedingungen abhängig (wir haben unterschiedliche Auffassungen darüber, was als ein unschwerwiegender und was als ein schwerwiegender Verstoß zu gelten hat) ist. 
Diese textstrukturellen Applikationen des Reizschemas ssozialer Stress stimmen ungefähr mit dem überein, was bereits seit einigen Jahrzehnten als /Witzı oder >Humor in Texten beschrieben wird. Neu ist allerdings die evolutionspsychologische Begründung und mit ihr die Konsequenz für die emotionale Bedeutung scherzhafter Dichtung: Denn das scherzhafte Verhalten eines literarischen Sprechers fungiert im Sinne der adaptiven Funktion >Kooperationsangebot< auch als appetitives Gegenüber, d.h. als ein sozialer Annäherungsreiz. Unsere Sympathie für den Erzähler der Buddenbrooks, für den Sprecher in Matthias Koeppels Starckdeutsch-Gedichten oder für den Soubrettentypus in zahlreichen Komödien ließe sich ohne Rekurs auf die adaptive Funktion des Lachens als soziales Signalverhalten im EEA nicht erklären. Lachen und der Grund für Lachen steuern maßgeblich sogenannte In-Group-/Out-Group-Effekte, wie sie zwischen Text und Leser stattfinden. Denselben Humor zu haben wie eine literarische Figur, bedeutet, sie auch zur eigenen sozialen Nahwelt zu zählen. Dies führt zu dem letzten Punkt, der unter evolutionärer Perspektive einige Erhellung gefunden hat: der Frage nach der (quasi)sozialen Bindung eines Lesers an Literatur (s. Voland 2007, 8 ff.).

\section{Soziale Bindung}

Humor ist nur ein Parameter, nach dem sich unsere Sympathie und unser Zugehörigkeitsgefühl in Bezug auf einen Autor, Erzähler oder eine Figur bemessen. Ausschlaggebend ist insbesondere auch die Übereinstimmung der moralischen Wertsysteme zwischen Rezipient und parasozialer Attrappe (vgl. das Konzept der »allegiance« bei Smith 1995). Diese mehr oder weniger zustimmende Haltung gegenüber parasozialen Attrappen ist oftmals Voraussetzung für intensivierte empathische Bemühungen und emotionale Reaktionen. Ich habe zu analytischen Zwecken vier Sympathiegrade differenziert, die für typische Reaktionen in Bezug auf literarische Attrappen in Anschlag gebracht werden können (Mellmann 2006a, 138-142): Das Spektrum reicht von der Sympathie für sirgendwies belebte und psychisch verfasste Wesen (1) über eine (meist ethisch begründete) Parteinahme (2) und freundschaftliche Familiarität (3) bis hin zum liebenden Erkennen eines reinzigartigen Gegenübers` (4).

Der erste Sympathiegrad erstreckt sich auch noch auf anthropomorphisierte Gegenstände und Tiere und erklärt, weshalb wir z.B. auch ein realistisch geschildertes Tier wie etwa in Marie von Ebner-Eschenbachs Krambambuli als Protagonisten einer Erzählung akzeptieren können. Die kognitiven Module zur Unterscheidung von belebten und unbelebten Objekten und zur Erkennung von intentionalem Verhalten (vgl. Jannidis 2007; Mellmann i.Vorb.) werden in der literarischen Attrappe fündig und schaffen die Voraussetzung dafür, dass unsere Fähigkeit zum »mind-reading« (Empathie) zum Einsatz kommt (Zunshine 2006, 
22) und wir den Zusammenhang der Handlung in ihrer Bedeutung für eine Figur verstehend mitvollziehen können.

Damit wir aber auch Mitleid mit dem Hund Krambambuli empfinden können, ist es nötig, dass wir sein Verhalten nicht nur nachvollziehbar finden, sondern im Großen und Ganzen auch billigen, d.h. ihn als einen Genossen derselben Wertegemeinschaft ansehen, der auch wir angehören. Dieser zweite Sympathiegrad involviert also meist ethisches Urteilsvermögen, kann aber auch auf anderen, stärker veräußerlichten Ähnlichkeitsrelationen (z.B. gleiche Herkunft) basieren (vgl. auch Eibl 2004, 170-174). Sympathie in diesem Sinne von Parteinahme ist die Voraussetzung für einen weiten Bereich dessen, was wir als ১Spannung $\prec$ bezeichnen: für das `Hoffen und Bangen mit einer Figur (Mellmann 2007, 257f., 263-265). Dies gilt für das Fußballspiel (das erst richtig Spaß macht, wenn man sich für eine Mannschaft entscheidet) genauso wie für die Unterscheidung von Protagonist und Antagonist in einem ethisch komplexen Roman.

Der dritte Sympathiegrad /Vertrautheit» wird vor allem durch eine umfassende Information über die fiktionale Figur vermittelt. Über wen wir so viel und so viel Intimes und Persönliches - wissen wie über einen Romanhelden, mit dem wir (gemessen an der Zeit der erzählten Welt) oftmals sein ganzes Leben verbringen<, der gehört nach den Gesetzen des EEA unbeirrbar zu unserer sozialen Nahwelt. Dieser Umstand mag erklären, warum es manchmal traurig ist, auf der letzten Seite eines Romans anzugelangen. Die Trauer, die der Romanleser beim Zuklappen des Buches empfindet, ist derjenigen ähnlich, die man empfindet, wenn man von einem guten Freund Abschied nehmen muss.

Dieser Effekt kann noch einmal gesteigert werden, wenn es sich nicht nur um sirgendwie netter und gut bekannte, sondern um Figuren handelt, die mit hohem Identifikationswert für ein höchst individuelles Problemempfinden ausgestattet sind. Das Beispiel des Wertherkults mag zeigen, wie Leser manchmal versuchen, den Kontakt mit einem sgeliebten fiktionalen Gegenüber durch wiederholte Lektüren und parareligiöse Riten weiter zu verlängern. Hier ist offensichtlich \Liebe im Sinne unserer emotionalen Adaptation für Partnerwahl (»Limerenz«, s. Mellmann 2006a, 141) angesprochen. Auch wurde festgestellt, dass die Rezeption eines Kunstwerks, wenn es als »schön« im emphatischen Sinne erfahren wird, generell solche liebesäquivalenten Wirkungen hervorrufen kann: Die Selbstvergessenheit während der stimuluskonzentrierten, sversunkenen Lektüresituation (Heller 2004, 246-248) und die Begeisterung für einen Autor, der alles so richtig und so schön dargestellt hat, sind dem Verhalten im Liebesaugenblick so ähnlich, dass `der Autor` oder `das Kunstwerkı als quasipersonales Liebesobjekt im Leser Gestalt gewinnen können. 


\title{
6. Beschluss
}

Die zurückliegenden Absätze geben einen groben Überblick über mögliche Korrelationen von Textmerkmalen und psychischen Gesetzmäßigkeiten, wie sie sich vom Standpunkt der evolutionären Psychologie aus erwarten lassen: Virtuelle Welten als Input für angeborene Mechanismen (Erfahrungsreize) sorgen generell für Lustempfinden und stiften damit eine Grundmotivation zum Lesen. Warum manche Reize zudem mit ınegativen`, manche mit spositiven Gefühlen beantwortet werden, lässt sich mit der evolutionären Bewährung eines vermeidenden bzw. annähernden Verhaltens den betreffenden Reizen gegenüber erklären. Auch abstrakter zu fassende, nicht mimetische, sondern strukturelle Textmerkmale wie die einer ggetroffenen Entscheidung oder eines sunschwerwiegenden Fehlers in der Autor-Leser-Kommunikation können in angeborenen Emotionsprogrammen (hier: Weinen und Lachen) >Treffer erzielen; entscheidend ist, wie abstrakt das jeweilige Auslöseschema verfasst ist. Hinzu kommen zahlreiche parasoziale Bindungen des Lesers an Autor und Figuren, die als Ansprechen sozialer Dispositionen auf anthropomorphe Vorstellungsinhalte begriffen werden können.

Das Verfahren der Korrelation von Reizqualität und psychischer Reaktion bedarf zum einen textanalytischer Kompetenzen, um die Reizkonfiguration im Text zu eruieren, und zum andern psychologischer Kompetenzen, die das vermutete Reizschema nebst dem zugehörigen psychischen Programm zu plausibilisieren erlauben. Das Verfahren dient dazu, erstens überhaupt emotional relevante Textmerkmale zu identifizieren und zweitens den Grad der »Schemakongruenz« (Mellmann 2006a, 52ff.), d.h. den Grad an Übereinstimmung zwischen der textuellen Reizkonfiguration und dem vermuteten emotionalen Auslöseschema, festzustellen. Eine biologische Literaturanalyse muss keineswegs in rreduktionistischeく Vereinfachungen führen, vielmehr lässt gerade eine differenzierte Analyse der Kongruenz von Texten mit biologisch verankerten Schemata wieder eine Unterscheidung von »Kitsch« und »Kunst« zu: Der billige tear jerker, die Boulevardkomödie, Abenteuer- und Liebesromane bieten fast ausschließlich >optimaler oder gar ssupernormale Attrappen, während das nach den Regeln der Abweichungs- oder Originalitätsästhetik gebaute `Kunstwerkı immer auch um Irritation und Verfremdung anzitierter Schemata bemüht ist und damit nicht zuletzt Raum gibt für die kulturelle Ausgestaltung und Neuprägung emotionaler Reaktionen.

\author{
Katja Mellmann \\ Institut für deutsche Philologie \\ Ludwig-Maximilians-Universität München
}




\section{Literatur}

Thomas Anz, Kulturtechniken der Emotionalisierung. Beobachtungen, Reflexionen und Vorschläge zur literaturwissenschaftlichen Gefühlsforschung, in: Karl Eibl/Katja Mellmann/Rüdiger Zymner (Hg.), Im Rücken der Kulturen, Paderborn 2007, 207-239. Niels Birbaumer/Robert F. Schmidt, Biologische Psychologie [1990], Berlin/Heidelberg ${ }^{4} 1999$. Brian Boyd, Evolutionary Theories of Art, in: Gottschall/Wilson 2005, 147-176. Joseph Carroll, Evolution and Literary Theory, Columbia 1995.

-, Literary Darwinism. Literature and the Human Animal, Columbia 2004.

Brett Cooke/Frederick Turner (Hg.), Biopoetics. Evolutionary Explorations in the Arts, Lexington 1999.

Leda Cosmides/John Tooby, Reasoning and Natural Selection, in: Renato Dulbecco (Hg.), Encyclopedia of Human Biology, Bd. 6, San Diego 1991, 493-503. Online verfügbar unter: <http://www.psych.ucsb.edu/research/cep/papers/Reasoning.pdf.>

-, Beyond Intuition and Instinct Blindness. The Case for an Evolutionarily Rigorous Cognitive Science, Cognition 50 (1994), 41-77. Online verfügbar unter: <http://www. psych.ucsb.edu/research/cep/papers/instinctblindess.pdf.>

-, Evolutionary Psychology and the Emotions, in: Michael Lewis/Jeannette M. HavilandJones (Hg.), Handbook of Emotions [1993], New York 22000, 91-115. Online verfügbar unter: <http://www.psych.ucsb.edu/research/cep/papers/Emotions2000.pdf.>

-, Evolutionary Psychology. Theoretical Foundations, in: Lynn Nadel (Hg.), Encyclopedia of Cognitive Science, London 2003, 54-64. Online verfügbar unter: <http://www. psych.ucsb.edu/research/cep/papers/A0529.pdf.>

Karl Eibl, Animal poeta. Bausteine der biologischen Kultur- und Literaturtheorie, Paderborn 2004.

-, Naturwissenschaft, in: Thomas Anz (Hg.), Handbuch Literaturwissenschaft, Bd. 2, Stuttgart/Weimar 2007, 486-495.

Dirk Eitzen, The Emotional Basis of Film Comedy, in: Carl Plantinga/Greg M. Smith (Hg.), Passionate Views. Film, Cognition, and Emotion, Baltimore/London 1999, 84-99.

Nico Frijda, The Nature of Pleasure, in: John A. Bargh/Deborah K. Apsley (Hg.), Unraveling the Complexities of Social Life, Washington 2000, 71-94.

Matthew Gervais/David Sloan Wilson, The Evolution and Functions of Laughter and Humor. A Synthetic Approach, The Quarterly Review of Biology 80 (2005), 395-430.

Jonathan Gottschall/David Sloane Wilson (Hg.), The Literary Animal. Evolution and the Nature of Narrative, Evanston 2005.

Agnes Heller, The Role of Emotions in the Reception of Artworks, in: Klaus Herding/ Bernhard Stumpfhaus (Hg.), Pathos, Affekt, Gefühl. Die Emotionen in den Künsten, Berlin 2004, 244-259.

Martin Huber, >Noch einmal mit Gefühk. Literaturwissenschaft und Emotion, in: Walter Erhard (Hg.), Grenzen der Germanistik. Rephilologisierung oder Erweiterung?, Stuttgart/Weimar 2004, 343-357.

Fotis Jannidis, Zur kommunikativen Intention. Anfänge, in: Karl Eibl/Katja Mellmann/ Rüdiger Zymner (Hg.), Im Rücken der Kulturen, Paderborn 2007, 185-204.

Gertrud Koch, Zu Tränen gerührt. Zur Erschütterung im Kino, in: Klaus Herding/Bernhard Stumpfhaus (Hg.), Pathos, Affekt, Gefühl. Die Emotionen in den Künsten, Berlin 2004, 562-574. 
Jerrold Levinson, Emotion in Response to Art, in: Mette Hjort/Sue Laver (Hg.), Emotion and the Arts, New York/Oxford 1997, 20-34.

Katja Mellmann, Emotionalisierung - Von der Nebenstundenpoesie zum Buch als Freund. Eine emotionspsychologische Analyse der Literatur der Aufklärungsepoche, Paderborn 2006 (Mellmann 2006a).

-, Literatur als emotionale Attrappe. Eine evolutionspsychologische Lösung des sparadox of fiction<, in: Uta Klein/Katja Mellmann/Steffanie Metzger (Hg.), Heuristiken der Literaturwissenschaft. Disziplinexterne Perspektiven auf Literatur, Paderborn 2006, 145-166 (Mellmann 2006b).

-, Vorschlag zu einer emotionspsychologischen Bestimmung von `Spannung‘, in: Karl Eibl/Katja Mellmann/Rüdiger Zymner (Hg.), Im Rücken der Kulturen, Paderborn 2007, 241-268.

-, Objects of »Empathy«. Characters (and Other Such Things) as Psycho-poetic Effects, in: Jens Eder/Fotis Jannidis/Ralf Schneider (Hg.), Characters in Fictional Worlds. Interdisciplinary Concepts [in Vorbereitung].

Winfried Menninghaus, Das Versprechen der Schönheit, Frankfurt a.M. 2003.

David S. Miall, An Evolutionary Framework for Literary Reading, in: Dick Schram/ Gerard Steen (Hg.), The Psychology and Sociology of Literature, Amsterdam/Philadelphia 2001, 407-419.

Gordon H. Orians/Judith H. Heerwagen, Evolved Responses to Landscapes, in: Jerome H. Barkow/Leda Cosmides/John Tooby (Hg.), The Adapted Mind. Evolutionary Psychology and the Generation of Culture, New York/Oxford 1992, 555-579.

Colin Radford, How Can We Be Moved by the Fate of Anna Karenina?, Proceedings of the Aristotelian Society, Suppl. 49 (1975), 67-80.

Rainer Schandry, Biologische Psychologie. Ein Lehrbuch, Weinheim/Basel 2003.

Klaus Scherer, Emotion Serves to Decouple Stimulus and Response, in: Paul Ekman/ Richard J. Davidson (Hg.), The Nature of Emotion. Fundamental Questions, New York/ Oxford 1994, 127-130.

Clemens Schwender, Medien und Emotionen. Evolutionspsychologische Bausteine einer Medientheorie [2001], Wiesbaden 22006.

Murray Smith, Engaging Characters. Fiction, Emotion, and the Cinema, Oxford 1995.

-, Wer hat Angst vor Charles Darwin? Die Filmkunst im Zeitalter der Evolution, in: Matthias Brütsch/Vinzenz Hediger/Ursula von Keitz/Alexandra Schneider/Margrit Tröhler (Hg.): Kinogefüble. Emotionalität und Film, Marburg 2005, 289-312.

Robert Storey, Mimesis and the Human Animal. On the Biogenetic Foundations of Literary Representation, Evanston 1996.

Ed Tan/Nico Frijda, Sentiment in Film Viewing, in: Carl Plantinga/Greg M. Smith (Hg.), Passionate Views. Film, Cognition, and Emotion, Baltimore/London 1999, 48-64.

Randy Thornhill, Darwinian Aesthetics, in: Charles Crawford/Dennis L. Krebs (Hg.), Handbook of Evolutionary Psychology. Ideas, Issues, and Applications, Mahwah/London 1998, 543-572. Wiederabgedruckt in: Voland/Grammar 2003, 9-35.

John Tooby/Leda Cosmides, The Past Explains the Present. Emotional Adaptations and the Structure of Ancestral Environments, Ethology and Sociobiology 11 (1990), 375424. Online verfügbar unter: <http://www.psych.ucsb.edu/research/cep/papers/ pastpresent1990.pdf.> 
-, The Psychological Foundations of Culture, in: Jerome H. Barkow/Leda Cosmides/ John Tooby (Hg.), The Adapted Mind. Evolutionary Psychology and the Generation of Culture, New York/Oxford 1992, 19-136. Online verfügbar unter: <http://www.psych.ucsb. edu/research/cep/papers/pfc92.pdf.>

-, Does Beauty Build Adapted Minds? Towards an Evolutionary Theory of Aesthetics, Fiction, and the Arts, SubStance 30:94/95 (2001), 6-27. Dt. Übers.: Schönheit und mentale Fitness. Auf dem Weg zu einer evolutionären Ästhetik, in: Uta Klein/ Katja Mellmann/Steffanie Metzger (Hg.), Heuristiken der Literaturwissenschaft. Disziplinexterne Perspektiven auf Literatur, Paderborn 2006, 217-243. Online verfügbar unter: <http://www.psych.ucsb.edu/research/cep/papers/beauty01.pdf.>

Eckart Voland, Virtuelle Welten in realen Gehirnen. Evolutionspsychologische Aspekte des Umgangs mit Medien, Zeitschrift für Literaturwissenschaft und Linguistik 146 (2007), 7-22.

Eckart Voland/Karl Grammar (Hg.), Evolutionary Aesthetics, Berlin 2003.

Lisa Zunshine, Why We Read Fiction. Theory of Mind and the Novel, Columbus 2006. 